Math. Model. Nat. Phenom.

Vol. 6, No. 7, 2011, pp. 82-99

DOI: $10.1051 / \mathrm{mmnp}: 20116707$

\title{
Vessel Wall Models for Simulation of Atherosclerotic Vascular Networks
}

\author{
Yu. Vassilevski ${ }^{11}$, S. Simakov ${ }^{2}$, V. Salamatova ${ }^{3}$, Yu. Ivanov ${ }^{3}$ and T. Dobroserdova ${ }^{4}$ \\ ${ }^{1}$ Institute of Numerical Mathematics RAS, \\ Gubkina st. 8, Moscow 119333, Russia \\ ${ }^{2}$ Moscow Institute of Physics and Technology, \\ Institutskyi Lane 9, Dolgoprudny 141700, Russia \\ ${ }^{3}$ Scientific Educational Center of Institute of Numerical Mathematics RAS, \\ Gubkina st. 8, Moscow 119333, Russia \\ ${ }^{4}$ Moscow State University, \\ Leninskie Gory, Moscow 119991, Russia
}

\begin{abstract}
There are two mathematical models of elastic walls of healthy and atherosclerotic blood vessels developed and studied. The models are included in a numerical model of global blood circulation via recovery of the vessel wall state equation. The joint model allows us to study the impact of arteries atherosclerotic disease of a set of arteries on regional haemodynamics.
\end{abstract}

Key words: atherosclerosis, mathematical modelling, blood flow, arterial wall, wall state equation AMS subject classification: 35Q92, 76Z05, 65M06, 65M25

\section{Introduction}

Vascular diseases are the main factors of mortality, and atherosclerosis is the most common vascular disease. Advanced medical treatment of atherosclerosis is one of the challenges for contemporary medicine. A study of vascular diseases and, in particular, of atherosclerosis impact on haemodynamics may be based on mathematical models and numerical simulation $[2,11,7,14,16,17]$. It is supposed to use the closed blood circulation model [14] in which the elastic properties of blood

\footnotetext{
${ }^{1}$ Corresponding author. E-mail: vasilevs@dodo.inm.ras.ru
} 
vessels are included in the wall state equation. The latter sets up the dependence of lumen cross section on the transmural pressure. Vessel diseases impact may be incorporated into the model through the recovery of the diseased vessel state equation. We suggest to derive the diseased wall state equation on the basis of the numerical solution of the equilibrium problem for a simplified elastic fiber-spring system which imitates the atherosclerotic vessel reaction to a deformation. The fiber-spring model was introduced in [17] where all materials were considered as linear.

In this paper we introduce approaches to modelling of atherosclerotic vessels elastic properties as nonlinear material ones. The key relationship in the theory of elasticity is the stress-strain relationship. Most bio-materials are nonlinear [4], arterial walls being the representative example. To review constitutive equations for arterial material we refer to $[5,6,18]$. We have applied a NeoHookean solid model which was used in modelling of atherosclerotic vessels [9] and aneurysms development study [19].

Two models of nonlinear elasticity were developed for healthy and diseased vessels. The first model is analytical and restricted by simple geometries of the vessel and the plaque. The second model is numerical and applies to much wider class of plaque geometry. We validate our numerical model via the analytical model and reproduce the state equation of the diseased wall. The recovered wall state equation allows us to employ the haemodynamic model and predict atherosclerotic plaques impact on the blood flow regime.

The outline of the paper is following. In Section 2 analytical thin-walled and numerical fiber models of a healthy artery are considered. Analytical three-layer and numerical fiber-spring models of atherosclerotic artery are presented in Section 3. Section 4 deals with the network blood flow model. In Section 5 the numerical results are presented. Section 6 sums up the paper.

\section{Elasticity models of healthy vessel}

This section presents two elastic models for a healthy vessel wall considered as Neo-Hookean material. The first model is based on equilibrium problem analytic solution for the thin-walled cylinder composed of Neo-Hookean material. Applicability of this approach is limited by vessels with straight cylindrical geometry. The second model uses a fiber representation of a vessel elastic wall. It benefits a simple finite difference discretization scheme and applicability to general geometries of blood vessels. In Section 5 we validate the fiber model via the reference solution obtained by the thin-wall model. The fiber model of a healthy vessel forms the basis for more complex atherosclerotic vessel model than presented in Section 3.

\subsection{Thin-wall model}

We consider a blood vessel as a thin-walled circular cylindrical shell inflated by the internal pressure. A strain in the axial direction is assumed to be negligible. The mechanical behavior of the arterial wall is defined by the incompressible Neo-Hookean material model. The Neo-Hookean 
solid is characterized by a strain energy density function $W$ which for the incompressible case is

$$
W=\frac{\mu}{2}\left(\lambda_{1}^{2}+\lambda_{2}^{2}+\lambda_{3}^{2}-3\right),
$$

where $\mu$ is the material constant, $\lambda_{i}$ are the principal stretches. For the incompressible NeoHookean material the relationship between the initial Young's modulus of the vessel (arterial) wall and the material constant $\mu$ is the following $\mu=E / 3$. The principal components of the Cauchy stress for the incompressible hyper-elastic material are given by

$$
\sigma_{i}=-p+\lambda_{i} \frac{\partial W}{\partial \lambda_{i}}, \quad i=1,2,3
$$

where $p$ is pressure to be determined.

In terms of the cylindrical polar coordinates $(R, \Theta, Z)$ the geometry of the tube is defined by $R_{0}-H / 2 \leq R \leq R_{0}+H / 2$, where $R_{0}$ and $H$ denote the middle radius and the thickness of the non-deformed tube, respectively.

The tube middle radius and thickness with respect to the axisymmetric deformed configuration are denoted by $r_{0}$ and $h$, respectively. In terms of cylindrical polar coordinates $(r, \theta, z)$ the tube under internal pressure $p_{0}$ is given by $r_{0}-h / 2 \leq r \leq r_{0}+h / 2$.

In our case the principal stretches are stretches in radial, circumferential and axial directions, i.e., $\lambda_{r}, \lambda_{\theta}$ and $\lambda_{z}$, respectively. They are defined by

$$
\lambda_{r}=h / H, \quad \lambda_{\theta}=r_{0} / R_{0},
$$

and $\lambda_{z}=1$ due to the assumption that the strain in the axial direction is negligible. Thus the incompressibility constraint is

$$
\lambda_{r} \lambda_{\theta} \lambda_{z}=\lambda_{r} \lambda_{\theta}=1 \text {. }
$$

Using the simplification $\sigma_{r}=-p+\lambda_{r} \partial W / \partial \lambda_{r}=0$ for the radial stress (the membrane approximation) we can find the pressure $p=\lambda_{r} \partial W / \partial \lambda_{r}=\mu \lambda_{r}^{2}$. The circumferential stress $\sigma_{\theta}$ is assumed to be approximately constant through the tube thickness; then balancing the tension in the tube and the inflation pressure we find that

$$
p_{0}=\frac{h \sigma_{\theta}}{r_{i}},
$$

where $r_{i}=r_{0}-h / 2$ is internal radius of the deformed cylinder and

$$
\sigma_{\theta}=\mu\left(\lambda_{\theta}^{2}-\lambda_{r}^{2}\right) .
$$

Taking into account (2.3), (2.4) and (2.5) we can write the following system of equations in two unknowns $\lambda_{\theta}$ and $\lambda_{r}$

$$
\left\{\begin{array}{l}
\lambda_{r} \lambda_{\theta}=1, \\
\mu\left(\lambda_{\theta}^{2}-\lambda_{r}^{2}\right)=\frac{p_{0}}{\lambda_{r} H}\left(\lambda_{\theta} R_{0}-\frac{\lambda_{r} H}{2}\right) .
\end{array}\right.
$$

System (2.7) reduces to a bi-quadratic equation on $\lambda_{\theta}$ (or $\lambda_{r}$ ) and thus may be solved analytically. Knowing solution of the system (2.7) we can find circumferential stress $\sigma_{\theta}$, the pressure $p$ and the geometrical parameters of the deformed tube $h$ and $R$. 


\subsection{Fiber model}

Using the approach described in [13], we imitate a response of the elastic surface to a deformation as the response of fibers collection to the same deformation. Let $\vec{X}(s, t)$ represent the fiber points position in space, where Lagrange coordinate $s$ is an arc length of the fiber in the unstressed state. We denote the fiber tension as $T(s, t)$. The local force density is given by the expression [15]:

$$
\vec{f}=\frac{\partial}{\partial s}(T \vec{\tau})
$$

where $\vec{\tau}$ is the unit tangent vector

$$
\vec{\tau}=\frac{\partial \vec{X}}{\partial s}\left|\frac{\partial \vec{X}}{\partial s}\right|^{-1} .
$$

In case of axisymmetric problem we can assume the middle surface of the cylindrical shell as a collection of independent ring fibers, and the fiber tension is defined by (2.6), i.e.

$$
T=\mu\left(\lambda_{\theta}^{2}-\lambda_{\theta}^{-2}\right), \lambda_{\theta}=\left|\frac{\partial X}{\partial s}\right|
$$

Substituting (2.8) with (2.10) results in final fiber-based stress-strain problem.

In case of Hookean wall material we use fiber tension in form [17]:

$$
T=E_{*}\left(\left|\frac{\partial X}{\partial s}\right|-1\right),
$$

where $E_{*}$ is the elastic modulus of the fiber.

We derive the numerical approximation of the fiber model following $[15,13]$. Let $N_{\theta}$ be the number of computational nodes on the fiber, $\vec{X}_{k}$ be the coordinates of the $k$ th node, $k=1, \ldots, N_{\theta}$, $\Delta s$ be the distance between neighboring nodes along the non-deformed fiber. We assume that $\Delta s$ will be the same for all fibers. In accordance with formulas (2.10), (2.11) we discretize $T$ and $\vec{\tau}$ :

$$
T_{k+1 / 2}=\mu\left(\left(\frac{\left|\vec{X}_{k+1}-\vec{X}_{k}\right|}{\Delta s}\right)^{2}-\left(\frac{\left|\vec{X}_{k+1}-\vec{X}_{k}\right|}{\Delta s}\right)^{-2}\right),
$$

and in case of linear tension:

$$
\begin{gathered}
T_{k+1 / 2}=E_{*}\left(\frac{\left|\vec{X}_{k+1}-\vec{X}_{k}\right|}{\Delta s}-1\right), \\
\vec{\tau}_{k+1 / 2}=\frac{\vec{X}_{k+1}-\vec{X}_{k}}{\left|\vec{X}_{k+1}-\vec{X}_{k}\right|} .
\end{gathered}
$$


The discrete elastic force at $k$ th node is defined as

$$
\vec{f}_{k}=\frac{T_{k+1 / 2} \vec{\tau}_{k+1 / 2}-T_{k-1 / 2} \vec{\tau}_{k-1 / 2}}{\Delta s}
$$

The above formulas set up our numerical fiber elastic model of the response to deformation.

\section{Elasticity models of atherosclerotic vessel}

Interior wall of an atherosclerotic vessel is covered by atherosclerotic plaques. Each plaque is composed of a thin fibrous cap and a lipid pool, and may cover lengthy parts of blood vessels. Mechanical studies show that besides the arterial wall, fibrous cap and lipid pool are composed by Neo-Hookean materials as well.

Following the strategy of Section 2 we present two elastic models for an atherosclerotic vessel with a lengthy plaque. The first model represents the diseased vessel as a three-layer circular cylindrical shell inflated by internal pressure. The internal and external layers are thin-walled cylindrical shells which represent the plaque fibrous cap and the vessel wall, respectively (see Fig. 1). The middle layer represents the lipid pool of the plaque. In contrast to [17] we assume that the materials forming all three layers are incompressible Neo-Hookean solids. This model is limited to cylindrical vessels with uniform and lengthy plaques. In this case the deformation problem reduces to a system of nonlinear equations which can be solved semi-analytically. The solution provides geometric characteristics of the equilibrium state for any internal pressure, and, in particular, the dependence of a lumen cross section on the transmural pressure.

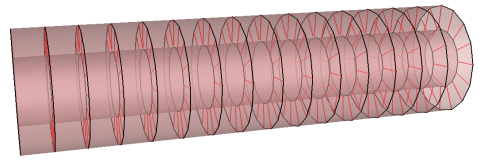

Figure 1: Geometry of the plaque.

The second model uses fiber representations of the arterial wall and the fibrous cap. The lipid pool is imitated by a set of radial springs with nonlinear relation between reaction force and displacement. An equilibrium state recovery of the above mentioned fiber-spring system is obtained in the framework of numerical approximation: finite difference discretization results in a system of nonlinear algebraic equations which has to be solved iteratively. This model benefits the wide class of plaque geometries. For instance, the Hookean materials fiber-spring model has been successfully tested for lengthy and local, symmetric and non-symmetric, plaques [17]. In Section 5 we validate the Non-Hookean materials fiber-spring model by solving the deformation problem of a vessel with a lengthy atherosclerotic plaque using the first three-layer model. 


\subsection{Equilibrium of thick-walled cylinder}

We consider a thick-walled cylinder under internal pressure $p_{a}$ and external pressure $p_{b}$. The deformation is defined by the mapping

$$
r=r(R), \quad \theta=\Theta, \quad z=Z .
$$

Then the principal stretches in radial, circumferential and axial directions, $\lambda_{r}, \lambda_{\theta}$ and $\lambda_{z}$ are

$$
\lambda_{r}=\frac{d r}{d R}, \quad \lambda_{\theta}=\frac{r}{R}, \quad \lambda_{z}=\frac{d z}{d Z}=1 .
$$

Using and integrating the incompressibility constraint $\lambda_{r} \lambda_{\theta} \lambda_{z}=1$ we can obtain

$$
r^{2}=R^{2}-K
$$

where $K$ is the constant of integration.

It has been shown [3] that the equilibrium equation along radial direction is

$$
R^{2} \frac{d W}{d r} d r=K \frac{d \sigma_{r}}{d r}
$$

which after integration gives

$$
p_{a}=p_{b}+\frac{1}{K} \int_{r_{a}}^{r_{b}} R^{2} \frac{d W}{d r} d r
$$

where $W$ is the stress energy density function defined by (2.1) for the cylinder and $W=W(r)$.

\subsection{Three-layer circular cylindrical shell}

The geometry of the non-deformed three-layer cylinder is defined by the following inequalities: $R_{1} \leq R \leq R_{2}$ defines the fibrous cap, $R_{2}<R \leq R_{3}$ defines the lipid pool, $R_{3}<R \leq R_{4}$ defines the arterial wall. The deformation is represented by mapping (3.1) and $r_{i}=r\left(R_{i}\right), i=1,2,3,4$. The three-layer cylinder is inflated by internal pressure $p_{0}$.

Then we can write equations describing the deformation of each layer of the three-layer cylindrical tube:

for the fibrous cap an equilibrium equation and an incompressibility constraint are expressed as

$$
\begin{aligned}
& \mu_{f}\left(\left(\lambda_{\theta}^{f}\right)^{2}-\left(\lambda_{r}^{f}\right)^{2}\right)=\frac{p_{0} r_{1}-p_{1} r_{2}}{r_{2}-r_{1}}, \\
& \lambda_{\theta}^{f} \lambda_{r}^{f}=1
\end{aligned}
$$

for the lipid pool an equilibrium equation is

$$
p_{1}=p_{2}+\frac{1}{K} \int_{r_{2}}^{r_{3}} R^{2} \frac{d W}{d r} d r
$$


for the arterial wall there is

$$
\begin{aligned}
& \mu_{a}\left(\left(\lambda_{\theta}^{a}\right)^{2}-\left(\lambda_{r}^{f}\right)^{2}\right)=\frac{p_{2} r_{3}}{r_{4}-r_{3}}, \\
& \lambda_{\theta}^{a} \lambda_{r}^{a}=1
\end{aligned}
$$

where $p_{1}$ and $p_{2}$ are contact pressure on surface $r=r_{2}$ and $r=r_{3}$, respectively; $\mu_{f}$ and $\mu_{a}$ are the material constants of the cap and the vessel wall, each of them being equal to corresponding initial Young's modulus divided by three $(\mu=E / 3) ; \lambda_{r}^{f}, \lambda_{\theta}^{f}$ and $\lambda_{r}^{a}, \lambda_{\theta}^{a}$ are the principal stretches in radial and circumferential directions for the fibrous cap and the arterial wall, respectively; $W$ is the strain energy density (2.1) which characterizes the lipid pool.

From continuity conditions of radial displacement and incompressibility constraints, we can find that

$$
\begin{aligned}
& r^{2}=R^{2}-K, \\
& \lambda_{\theta}^{f}=\frac{r_{1}+r_{2}}{R_{1}+R_{2}}=\frac{\sqrt{R_{1}^{2}-K}+\sqrt{R_{2}^{2}-K}}{R_{1}+R_{2}}, \\
& \lambda_{\theta}^{a}=\frac{r_{3}+r_{4}}{R_{3}+R_{4}}=\frac{\sqrt{R_{3}^{2}-K}+\sqrt{R_{4}^{2}-K}}{R_{3}+R_{4}} .
\end{aligned}
$$

Thus we can write system of equations describing the deformation of the three-layer cylindrical tube under internal pressure $p_{0}$ :

$$
\left\{\begin{array}{l}
\mu_{f}\left(\left(\lambda_{\theta}^{f}\right)^{2}-\left(\lambda_{\theta}^{f}\right)^{-2}\right)=\frac{p_{0} r_{1}-p_{1} r_{2}}{r_{2}-r_{1}}, \\
p_{1}=p_{2}+\frac{1}{K} \int_{r_{2}}^{r_{3}} R^{2} \frac{d W}{d r} d r \\
\mu_{a}\left(\left(\lambda_{\theta}^{a}\right)^{2}-\left(\lambda_{\theta}^{a}\right)^{-2}\right)=\frac{p_{2} r_{3}}{r_{4}-r_{3}},
\end{array}\right.
$$

where $r(R), \lambda_{\theta}^{f}, \lambda_{\theta}^{a}$ are defined by (3.9) and the system (3.10) is the system of nonlinear equations with three unknowns $p_{1}, p_{2}$, and $K$. The solution of this system defines each layer principal stretches and may be obtained by conventional software (e.g. MAPLE).

\subsection{Fiber-spring model}

We use a fiber model for the fibrous cap and the arterial wall. An elastic model of the lipid pool is based on its representation by a set of radial springs. In order to estimate spring displacement, we use a solution of the deformation problem for the incompressible isotropic cylinder $(a \leq r \leq b)$ under internal pressure $p_{a}$ and external pressure $p_{b}$. Since we work within the normal physiological range of blood pressure, we solve the deformation problem using the hypothesis of linear elasticity theory. In this case the relation between the radial displacement $u(r)$ of cylinder's points and given pressures is expressed as

$$
p_{a}-p_{b}=\frac{2\left(b^{2}-a^{2}\right) E_{c} r}{3 a^{2} b^{2}} u(r)
$$


where $E_{c}$ is Young's modulus of the cylinder.

For each radial spring, displacements of its end points are derived on the basis of (3.11) and the assumption that only radial displacements may occur. The unknown fields of fibrous cap and arterial wall radial displacements are specified by $u^{a}(\theta, z)$ and $u^{b}(\theta, z)$, respectively.

Balancing forces on the external (artery) and internal (fibrous cap) layers implies

$$
\begin{aligned}
& p_{a}=p_{0}-\left(\vec{f}^{\mathrm{cap}}, \vec{n}^{\mathrm{cap}}\right) h^{\text {cap }}, \\
& p_{b}=\left(\vec{f}^{\mathrm{art}}, \vec{n}^{\mathrm{art}}\right) h^{\mathrm{art}},
\end{aligned}
$$

where $\vec{f} \vec{f}^{\text {art }}, \vec{n}^{\text {art }}, h^{\text {art }}$ and $\vec{f}^{\text {cap }}, \vec{n}^{\text {cap }}, h^{\text {cap }}$ are force density, surface normal, layer thickness of the artery wall and the fibrous cap, respectively. Similarly to radial displacements $u^{a}(\theta, z), u^{b}(\theta, z)$, the forces, normals and thicknesses are functions of coordinates $\theta, z$. Moreover, due to (2.8) and (2.4) these functions depend on radial displacements as well:

$$
\begin{gathered}
\vec{f}^{\text {cap }}=\vec{f}\left(a+u^{a}(\theta, z), \theta, z\right), \quad \vec{n}^{\text {cap }}=\vec{n}^{\text {cap }}\left(a+u^{a}(\theta, z), \theta, z\right), \quad h^{\text {cap }}=H^{\text {cap }} a /\left(a+u^{a}\right), \\
\vec{f}^{\text {art }}=\vec{f}\left(b+u^{b}(\theta, z), \theta, z\right), \quad \vec{n}^{\text {art }}=\vec{n}^{\text {art }}\left(b+u^{b}(\theta, z), \theta, z\right), \quad h^{\text {art }}=H^{\text {art }} b /\left(b+u^{b}\right),
\end{gathered}
$$

where $\vec{f}$ is given by (2.8), $H^{\text {cap }}$ and $H^{\text {art }}$ are thicknesses of fibrous cap and artery wall in nonstressed state.

In accordance with (3.12), (3.13) and (3.11) for $r=a$ and $r=b$, the radial displacements fields satisfy the following equations:

$$
\left\{\begin{array}{l}
\left(\vec{f}^{\text {art }}, \vec{n}^{\text {art }}\right) h^{\text {art }}+\left(\vec{f}^{\text {cap }}, \vec{n}^{\text {cap }}\right) h^{\text {cap }}-p_{0}=u^{a} 2\left(b^{2}-a^{2}\right) E_{c} / 3 a b^{2}, \\
\left(\vec{f}^{\text {art }}, \vec{n}^{\text {art }}\right) h^{\text {art }}+\left(\vec{f}^{\text {cap }}, \vec{n}^{\text {cap }}\right) h^{\text {cap }}-p_{0}=u^{b} 2\left(b^{2}-a^{2}\right) E_{c} / 3 a^{2} b .
\end{array}\right.
$$

The numerical model is based on equidistant $N_{z}$ pairs of concentric ring fibers with coordinates $z_{i}, i=1, \ldots, N_{z}$, discretized by the same number of grid nodes with uniform angular distribution $\theta_{k}, k=1, \ldots, N_{\theta}$. The discretized system of equations (3.16) reads as

$$
\left\{\begin{array}{l}
\left(\vec{f}_{i k}^{\text {art }}, \vec{n}_{i k}^{\text {art }}\right) h_{i k}^{\text {art }}+\left(\vec{f}_{i k}^{\text {cap }}, \vec{n}_{i k}^{\text {cap }}\right) h_{i k}^{\text {cap }}-u_{i k}^{a} 2\left(b^{2}-a^{2}\right) E_{c} a / 3 a b^{2}=p_{0}, \\
\left(\vec{f}_{i k}^{\text {art }}, \vec{n}_{i k}^{\text {art }}\right) h_{i k}^{\text {art }}+\left(\vec{f}_{i k}^{\text {cap }}, \vec{n}_{i k}^{\text {cap }}\right) h_{i k}^{\text {cap }}-u_{i k}^{b} 2\left(b^{2}-a^{2}\right) E_{c} b / 3 a^{2} b=p_{0},
\end{array}\right.
$$

where

$$
\begin{aligned}
\vec{f}_{i k}^{\text {cap }} & =\vec{f}\left(a+u_{i k}^{a}, \theta_{k}, z_{i}\right), \\
\vec{f}_{i k}^{\text {art }} & =\vec{f}\left(b+u_{i k}^{b}, \theta_{k}, z_{i}\right), \\
\vec{n}_{i k}^{\text {cap }} & =\vec{n}\left(a+u_{i k}^{a}, \theta_{k}, z_{i}\right), \\
\vec{n}_{i k}^{\text {art }} & =\vec{n}\left(b+u_{i k}^{b}, \theta_{k}, z_{i}\right), \\
h_{i k}^{\text {cap }} & =\frac{H^{\text {cap }} a}{a+u_{i k}^{a}}, \\
h_{i k}^{\text {art }} & =\frac{H^{\text {art }} b}{b+u_{i k}^{b}} .
\end{aligned}
$$


The system of $2 N_{z} N_{\theta}$ nonlinear equations (3.17) with $2 N_{z} N_{\theta}$ unknowns $u_{i k}^{a}, u_{i k}^{b}$ is solved by the Inexact Newton-Krylov method.

\section{Network blood flow}

We study atherosclerosis impact on the regional blood circulation using the closed circulation model [14]. In this section we briefly outline the model and discuss its functionality for vascular diseases simulation.

Blood flow is considered as a pulsating flow of incompressible fluid streaming through the network of elastic tubes (vessels). For every elastic tube (vessel) we account mass and momentum change by means of hyperbolic equations written in a characteristic form:

$$
\begin{aligned}
\partial S / \partial t+\partial(S u) / \partial x & =0 \\
\partial u / \partial t+\partial\left(u^{2} / 2+p / \rho\right) / \partial x & =-16 \mu u \eta(\hat{S}) / \hat{S}
\end{aligned}
$$

where $t$ is a time, $x$ is a coordinate along the vessel, $\rho$ is a blood density, $S(t, x)$ is a vessel cross section area, $u(t, x)$ is linear flow velocity averaged over the vessel area at coordinate $x$, where $p$ is pressure (with respect to the atmospheric pressure), function $\eta$ is set as

$$
\eta(\hat{S})= \begin{cases}2, & \hat{S} \geqslant 1, \\ \hat{S}+\frac{1}{\hat{S}}, & S<1 .\end{cases}
$$

Here $\hat{S}=S / \bar{S}, \bar{S}$ is cross section area under zero transmural pressure $p(S)-p_{*}$ and zero velocity, $p_{*}$ is a pressure in the surrounding tissues, $\mu$ is a friction factor.

The integration domain for (4.1), (4.2) is a set of 1D elastic tubes connected in a closed network according to the vascular anatomy. The structure of the systemic arterial network is shown in Fig. 2. The details for the methods of the closed vascular network $1 \mathrm{D}$ structure reconstruction and it's parameters identification are given in [14]. The presented model is a global circulation model.

The vessels are connected with each other at the nodes and with the heart inlets/outlets through the boundary conditions set that is formed by Poiseuille's pressure drop conditions, mass balance equation combined with the appropriate compatibility condition for (4.1),(4.2):

$$
\begin{gathered}
p_{k}\left(S_{k}\left(t, \tilde{x}_{k}\right)\right)-p_{\text {node }}^{l}(t)=\varepsilon_{k} R_{k}^{l} S_{k}\left(t, \tilde{x}_{k}\right) u_{k}\left(t, \tilde{x}_{k}\right), \quad k=k_{1}, k_{2}, \ldots, k_{M}, \\
\sum_{k=k_{1}, k_{2}, \ldots, k_{M}} \varepsilon_{k} S_{k}\left(t, \tilde{x}_{k}\right) u_{k}\left(t, \tilde{x}_{k}\right)=0,
\end{gathered}
$$

where $l$ is a node index, $k$ is a vessel index, $k_{1}, k_{2}, \ldots, k_{M}$ and $M$ are indexes and number of the vessels meeting at the node; $p_{\text {node }}^{l}(t)$ is a pressure at the vessels junction point; $R_{k}^{l}$ is a hydraulic resistance for the flow from the $k$-th vessel to the $l$-th node. For the vessels incoming into a node we set $\varepsilon_{k}=1, \tilde{x}_{k}=L_{k}$, for the outgoing vessels we set $\varepsilon_{k}=-1, \tilde{x}_{k}=0$. At the heart junction 


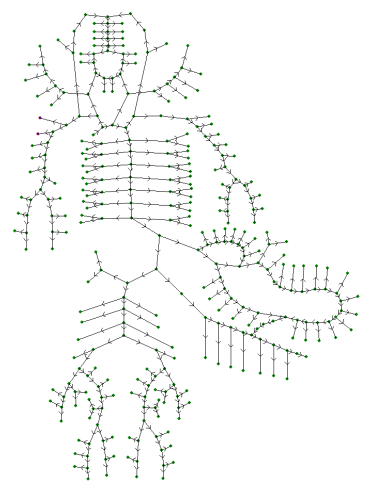

Figure 2: Systemic arterial network structure.

nodes the product $S_{k}(t, x) u_{k}(t, x)$ in (4.4), (4.5) is replaced by the volumetric flow to/from the chamber.

The vessel wall elastic properties are included in (4.1), (4.2) by the wall state equation giving the dependence of transmural pressure $p-p_{*}$ on the vessel cross section area $S$ :

$$
p(S)-p_{*}=\rho c_{0}^{2} f(S),
$$

where $c_{0}$ is the rate of small disturbance propagation in the vessel wall. For healthy vessels we use $S$-shaped function $f[14]$ :

$$
f(S)= \begin{cases}\exp (S / \bar{S}-1)-1, & S>\bar{S} \\ \ln (S / \bar{S}), & S \leqslant \bar{S},\end{cases}
$$

which seems to be a feasible approximation of the experimental curves.

In general, function $f(S)$ depends on the type and state of the vessel wall and external factors: elastic or muscular type of the wall, installed endovascular devices, atherosclerotic plaque, occlusion, etc. It is difficult to estimate analytical approximation $f(S)$ for the above mentioned cases. In general, the wall state equation should be recovered in the mechanical laboratory study. We suggest to replace the expensive and time consuming experimental study with the vessel wall numerical models. The models apply to Hookean or Neo-Hookean elastic materials and provide discretized functional dependence (4.7). Similar approach with Hookean models have been developed in $[16,17]$.

For every vessel, the equations (4.1)-(4.7) are solved by the hybrid (first and second order) explicit grid-characteristic method. This model also includes a set of stiff ODEs which describes the heart functioning in terms of volume averaged model [14]. The system of stiff ODEs is solved by A- and L-stable implicit third order Runge-Kutta method. 


\section{Results}

Presentation of the numerical results have been divided into three parts.

In the first part we have studied our numerical fiber and fiber-spring models. Firstly, we have considered the healthy common carotid artery and validate the numerical fiber model via the analytical thin-walled model by comparing their wall state equations. Secondly, we analyze the lengthy atherosclerotic plaque on the common carotid artery with different lumens and validate the numerical fiber-spring model via the analytical three-layer model. These comparisons demonstrate acceptable errors of our numerical models and thus verify the presented approach.

In the second part we have studied the difference between Hookean and Neo-Hookean material models by comparing the discretized wall state equations for healthy and atherosclerotic artery.

The third part have demonstrated practical advantages of the developed elasticity models. These models are incorporated in the network blood flow model via the discretized wall state equations. We chose a region of the common carotid artery as an example of the atherosclerotic network.

\subsection{Validation of fiber and fiber-spring models}

Here we consider a straight cylindrical vessel and compare the analytical thin-wall model and the numerical fiber models. The wall material is assumed to be Neo-Hookean. The solution of the static equilibrium problem provides the dependence of cross sectional area $S$ on the pressure load $p_{0}$. Values of Young's modulus, an inner radius and a thickness of the arterial wall have been chosen according to the values known for the common carotid artery: $E=10^{6} \mathrm{~Pa}$ [12], $R=0.45 \mathrm{~cm}$ and $H=0.07 \mathrm{~cm}$, respectively. The computed pressure to relative cross section relationship is summarized in Table 1 where $S_{0}$ is an area of the healthy unloaded artery. We observe the error less than $1 \%$ for the whole range of pressures and less than $0.2 \%$ for pressures not exceeding $8 \mathrm{kPa}$. As we see later, the maximum blood pressure for the common carotid simulations is limited by this value.

Similarly we compare the solutions of the static equilibrium problem obtained by the analytical three-layer model and the numerical fiber-spring model of an atherosclerotic vessel. The lumen is set to $50 \%, 30 \%$ and $10 \%$. The elastic constants and geometric characteristics correspond to the common carotid artery: Young's moduli for the arterial wall $E_{\text {art }}=10^{6} \mathrm{~Pa}$ [12], the fibrous cap $E_{\text {cap }}=5 \cdot 10^{5} \mathrm{~Pa}$ [8] and the lipid pool $E_{\text {pool }}=10^{3} \mathrm{~Pa}$ [1], the thickness of the fibrous cap and the arterial wall $H_{\text {art }}=H_{\text {cap }}=0.07 \mathrm{~cm}$. The computed pressure to relative cross section relationship is summarized in Table 2. We have obtained the error less than $2 \%$ in the whole range of pressures observed in the common carotid artery.

\subsection{Elasticity models for Hookean and Neo-Hookean materials}

In $[16,17]$ we have used the Hookean (linear) material model in order to study the impact of elastic vessel properties changes due to the endovascular filter implantation or the atherosclerotic plaque occurrence. In this paper, we use the Neo-Hookean (nonlinear) material model for both 


\begin{tabular}{|c|c|c|c|}
\hline & \multicolumn{2}{|c|}{$S / S_{0}$} & \\
\hline$p_{0}, \mathrm{kPa}$ & $A$ & $B$ & error,\% \\
\hline 0 & 1 & 0.9984 & 0.16 \\
1 & 1.0113 & 1.0105 & 0.08 \\
2 & 1.0230 & 1.0229 & 0.01 \\
3 & 1.0350 & 1.0355 & 0.05 \\
4 & 1.0475 & 1.0484 & 0.09 \\
5 & 1.0604 & 1.0616 & 0.12 \\
6 & 1.0737 & 1.0751 & 0.13 \\
7 & 1.0875 & 1.0889 & 0.13 \\
8 & 1.1018 & 1.1030 & 0.11 \\
9 & 1.1166 & 1.1173 & 0.07 \\
10 & 1.1320 & 1.1321 & 0.01 \\
11 & 1.1480 & 1.1471 & 0.07 \\
12 & 1.1646 & 1.1625 & 0.18 \\
13 & 1.1819 & 1.1782 & 0.31 \\
14 & 1.1999 & 1.1943 & 0.47 \\
\hline
\end{tabular}

Table 1: Relative cross section of the common carotid artery under static pressure load $p_{0}$ obtained by analytical thin-walled model (A) and numerical fiber model (B).

healthy and atherosclerotic arteries. In this section we compare both material models in terms of the computed wall state equation in order to examine the difference between the two material models. Figure 3 demonstrates the dependencies $p_{0}\left(S / S_{0}\right)$ for the cases of healthy vessel (left panel) and atherosclerotic vessel with a lengthy plaque (right panel) and $10 \%$ lumen. Parameters for the static equilibrium problem are set according to the common carotid artery properties.

In case of the healthy artery, we observe substantial deviation of the Hookean and Neo-Hookean curves $p_{0}\left(S / S_{0}\right)$ for pressures higher than $8 \mathrm{kPa}$. Since such high pressure is achieved no longer than 0.1 seconds within the cardiac cycle, the discrepancy impact in the wall state equations is almost negligible.

For the atherosclerotic artery, the discrepancy in the wall state equations is more distinct. The substantial pressures difference is observed for transmural pressures over $4 \mathrm{kPa}$ as well as for negative transmural pressures. It results in higher sensitivity to inflating transmural pressure and higher resistivity to deflating transmural pressure.

\subsection{Blood flow in atherosclerotic vessels network}

We have used the computed wall state equations (see Tables 1,2) for atherosclerotic arteries in the network blood flow model. The following scenario of atherosclerosis has been chosen: the common, internal and external carotid arteries (vessels No. 9, 65, 66 in Fig. 4) are healthy or diseased with lengthy plaques and lumens are $50 \%$ or $30 \%$ whereas the other vessels are considered 


\begin{tabular}{|c|c|c|c|c|c|c|c|c|c|}
\hline & \multicolumn{3}{|c|}{$50 \%$ lumen } & \multicolumn{3}{|c|}{$30 \%$ lumen } & \multicolumn{3}{|c|}{$10 \%$ lumen } \\
\hline & \multicolumn{2}{|c|}{$S / S_{0}$} & \multirow[b]{2}{*}{ error, $\%$} & \multicolumn{2}{|c|}{$S / S_{0}$} & \multirow[b]{2}{*}{ error, $\%$} & \multicolumn{2}{|c|}{$S / S_{0}$} & \multirow[b]{2}{*}{ error, $\%$} \\
\hline$p_{0}, \mathrm{kPa}$ & A & B & & A & B & & $\mathrm{A}$ & B & \\
\hline 0 & 0.5000 & 0.4986 & 0.27 & 0.3000 & 0.2995 & 0.16 & 0.1000 & 0.0998 & 0.25 \\
\hline 1 & 0.5044 & 0.5041 & 0.07 & 0.3028 & 0.3027 & 0.03 & 0.1008 & 0.1009 & 0.04 \\
\hline 2 & 0.5089 & 0.5090 & 0.02 & 0.3056 & 0.3059 & 0.09 & 0.1016 & 0.1019 & 0.22 \\
\hline 3 & 0.5134 & 0.5140 & 0.10 & 0.3085 & 0.3092 & 0.20 & 0.1025 & 0.1029 & 0.41 \\
\hline 4 & 0.5181 & 0.5190 & 0.18 & 0.3115 & 0.3125 & 0.31 & 0.1034 & 0.1040 & 0.58 \\
\hline 5 & 0.5228 & 0.5241 & 0.25 & 0.3145 & 0.3158 & 0.41 & 0.1042 & 0.1050 & 0.76 \\
\hline 6 & 0.5277 & 0.5293 & 0.31 & 0.3176 & 0.3192 & 0.51 & 0.1051 & 0.1061 & 0.92 \\
\hline 7 & 0.5326 & 0.5346 & 0.37 & 0.3208 & 0.3227 & 0.60 & 0.1060 & 0.1072 & 1.09 \\
\hline 8 & 0.5376 & 0.5399 & 0.42 & 0.3240 & 0.3262 & 0.68 & 0.1070 & 0.1083 & 1.24 \\
\hline 9 & 0.5428 & 0.5453 & 0.47 & 0.3273 & 0.3297 & 0.75 & 0.1079 & 0.1094 & 1.39 \\
\hline 10 & 0.5480 & 0.5508 & 0.51 & 0.3306 & 0.3333 & 0.82 & 0.1089 & 0.1106 & 1.54 \\
\hline 11 & 0.5534 & 0.5564 & 0.54 & 0.3341 & 0.3370 & 0.88 & 0.1099 & 0.1117 & 1.67 \\
\hline 12 & 0.5589 & 0.5621 & 0.57 & 0.3376 & 0.3407 & 0.94 & 0.1109 & 0.1129 & 1.81 \\
\hline 13 & 0.5645 & 0.5678 & 0.59 & 0.3411 & 0.3445 & 0.98 & 0.1119 & 0.1141 & 1.93 \\
\hline
\end{tabular}

Table 2: Relative cross section of the atherosclerotic common carotid artery with lumen 50\%, 30\% and $10 \%$ under static pressure $p_{0}$ obtained by the analytical three-layer model (A) and numerical fiber-spring model (B).
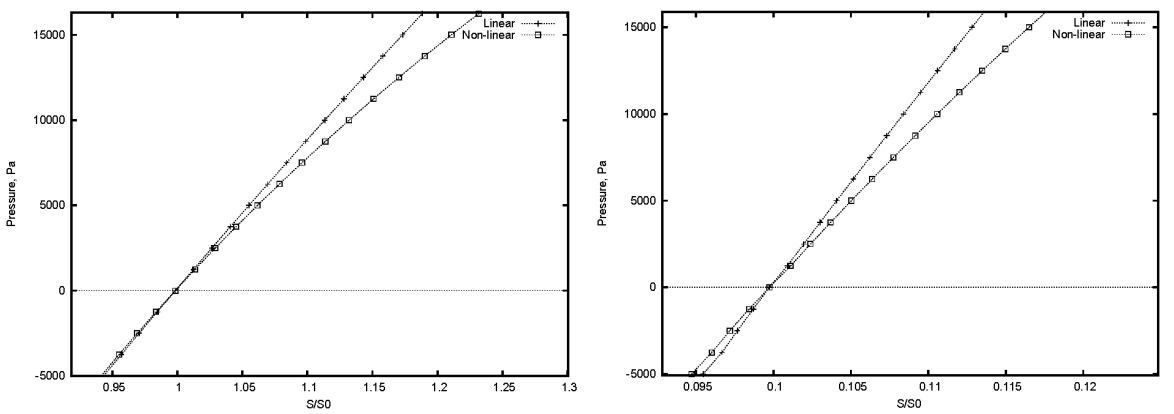

Figure 3: Comparison of wall state equations for Hookean and Neo-Hookean materials: healthy artery (left), atherosclerotic artery (right). 
to be healthy. For the sake of brevity we consider a healthy vessel as an atherosclerotic vessel with $100 \%$ lumen. The wall state equation for vessels No. 9, 65, 66 is computed on the basis of the fiber-spring model for Neo-Hookean materials. The wall state equation for the other vessels is defined by analytical formulas (4.6), (4.7).
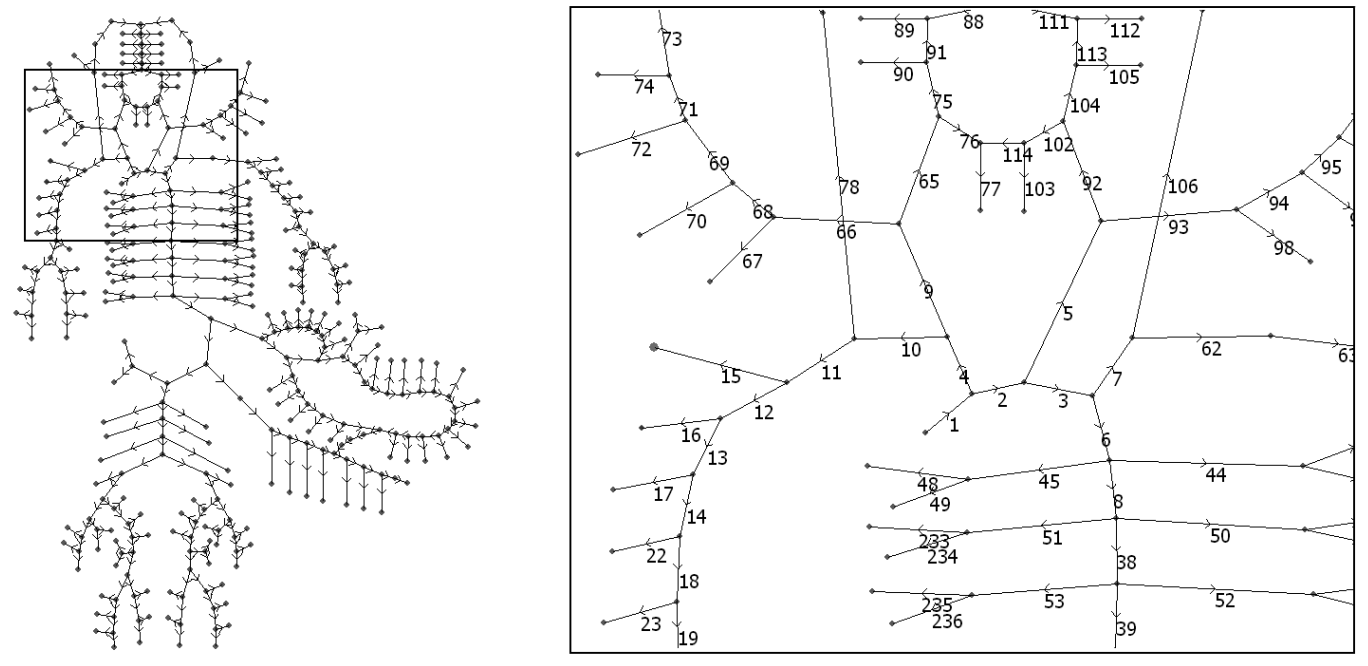

Figure 4: Fragment of the systemic arterial network.

In the first series of the experiments (see Figs. 5, 6, 7, 8) we assume that the right common carotid artery is damaged by the lengthy atherosclerotic plaque with $30 \%, 50 \%$ and $100 \%$ lumen. The pressure and velocity profiles in common (No. 9), subsequent internal (No. 65) and external (No. 66) carotid arteries are shown in Figs. 5, 6. The pressure and velocity profiles in the brachial artery (No. 12), external carotid continuation (No. 68) and the artery of the Willis circle (No. 91) are shown in Figs. 7, 8.

The left panel of Fig. 5 reveals no substantial changes of the pressure profile in the common carotid artery. The atherosclerosis affects only the velocity profile resulting in two-fold decrease of the maximum velocity under $30 \%$ lumen (see left panel of Fig. 6). This observation is confirmed by the well-known fact that haemodinamically noticeable atherosclerosis or stenosis has lumen less than $50 \%$. The pressure and velocity profiles of the internal and external carotid arteries are affected much more noticeable (central and right panels of Figs. 5, 6). They reveal two-fold decrease of pressure and velocity maximums for the $30 \%$ lumen. The network blood flow model allows us to trace this effect throughout the entire network. For instance, considerable changes in vessels No. 68 and No. 91 (see Figs. 7, 8) may be interpreted as initial stages of oxygen deficiency in such important parts as eye and brain. The pressure and velocity decrease in small vessels leads to thrombosis and subsequent sharp pressure increase. For the eye and the brain it results in ischemia, hemorrhage, blindness and stroke. These well known atherosclerosis consequences are also confirmed by the proposed mathematical model. The analysis of the collateral route (vessels 
No. 5, No. 92, No. 93 etc.) reveals no substantial changes in haemodynamics and is not presented here.

In the second series of experiments we consider simultaneous atherosclerotic disease of vessels No. 9, 65, 66 with 50\%, 50\%, 50\% lumens and with 50\%, 30\%, 30\% lumens, respectively. The numerical results are qualitatively similar to those discussed above and thus are not presented here in detail. We notice the impact of the assumed atherosclerosis on the vessels No. 68 and No. 91: it is less pronounced than the impact of the single atherosclerotic vessel (the common carotid artery) with 30\% lumen. We explain this fact by smoothing pressure and velocity profiles along the route in case of the simultaneous atherosclerotic disease.
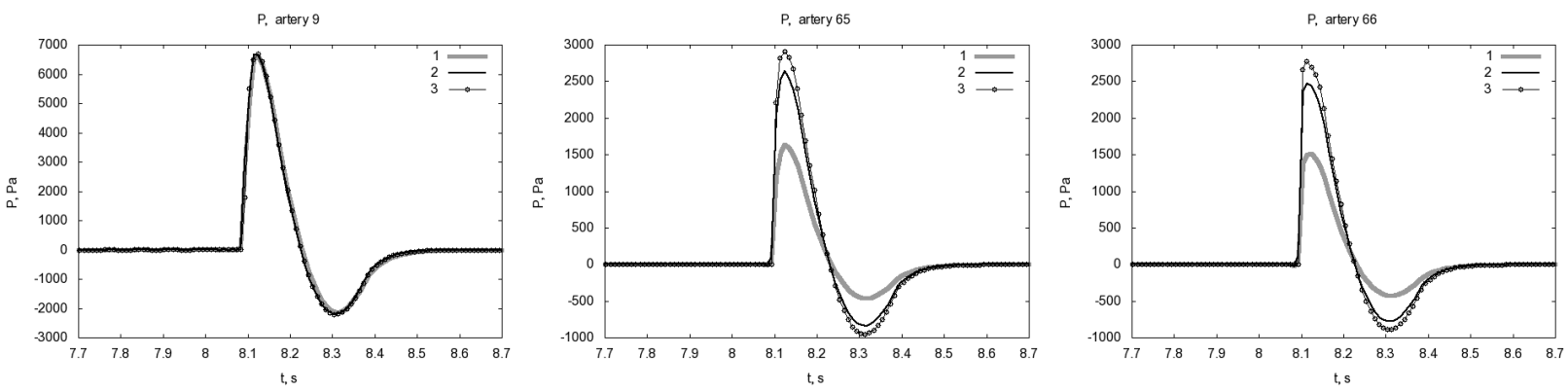

Figure 5: Pressure, Pa. 1 - 30\% lumen, $2-50 \%$ lumen, 3 - healthy vessel.
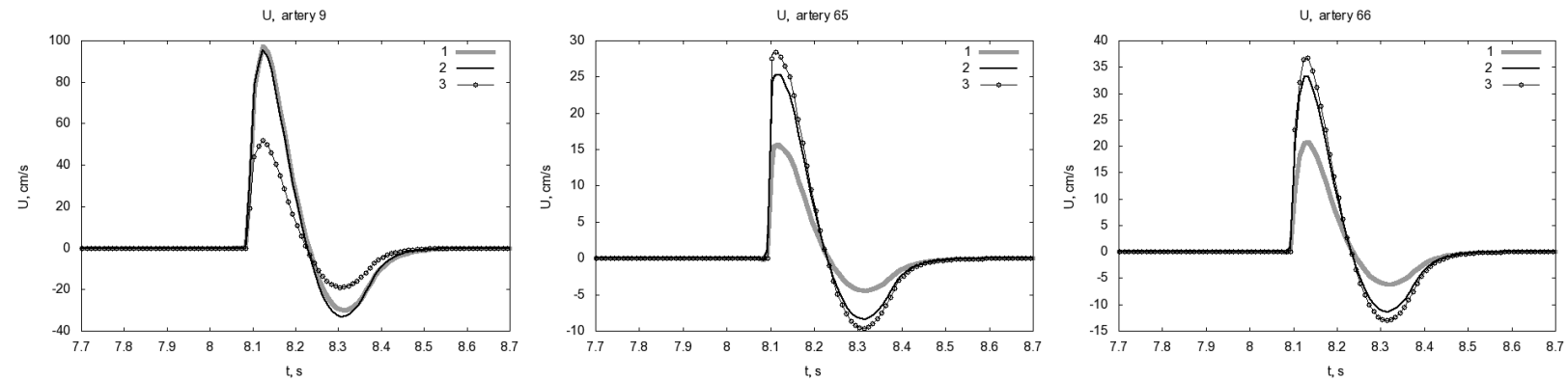

Figure 6: Velocity, cm/s. $1-30 \%$ lumen, $2-50 \%$ lumen, 3 - healthy vessel.

\section{Conclusions}

Most of the biological soft tissues demonstrate nonlinear strain-stress relationships, in particular, at high stresses. Atherosclerotic arteries elastic properties can be represented by the three-layer composition of Neo-Hookean materials. In this paper we considered lengthy uniform atherosclerotic plaques in straight vessels and introduced the numerical fiber-spring model of atherosclerotic artery wall which accounts its three-layer Neo-Hookean structure. In our recent paper [17], we 

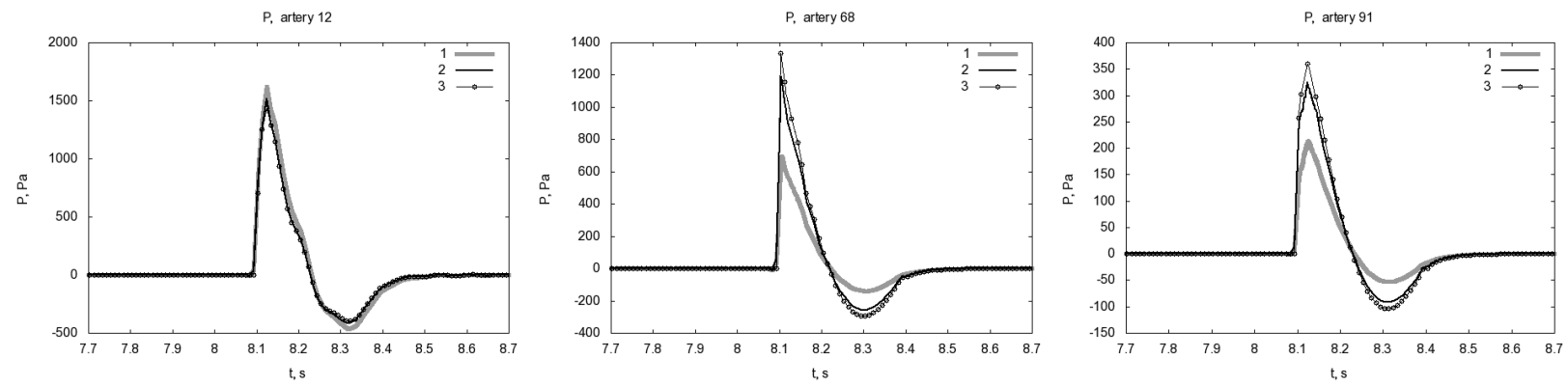

Figure 7: Pressure, Pa. 1 - 30\% lumen, 2 - 50\% lumen, 3 - healthy vessel.
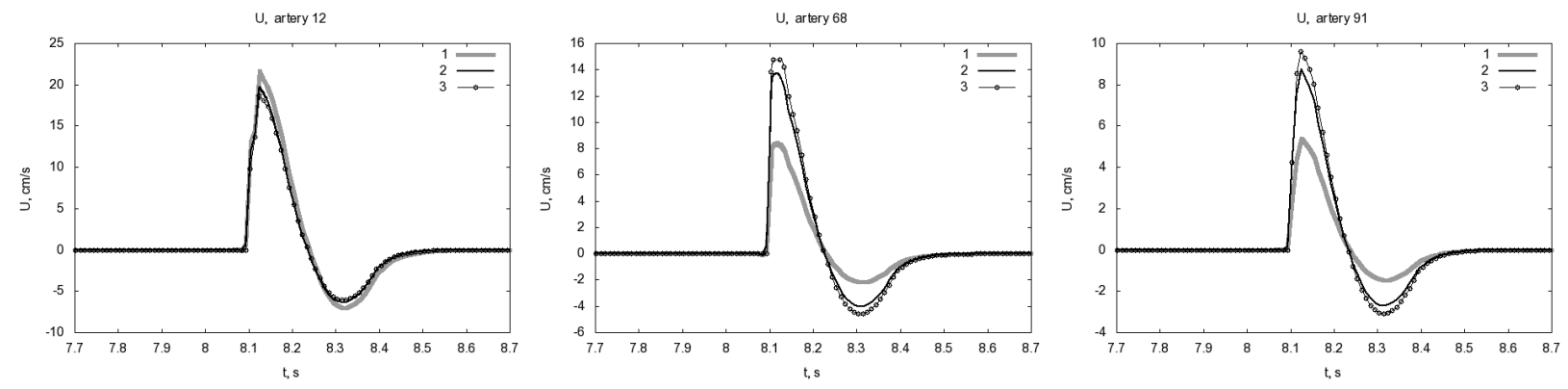

Figure 8: Velocity, cm/s. 1 - 30\% lumen, $2-50 \%$ lumen, 3 - healthy vessel.

studied atherosclerotic artery wall as a composition of Hookean materials and showed that the numerical fiber-spring model could be applied to general shaped atherosclerotic plaques. In both papers, the elastic vessel wall numerical model produces the pressure to cross section relationship, or the wall state equation, which is the input function in the global network blood flow model [14]. The latter allows us to study the atherosclerotic disease impact on haemodynamics. Further development of this approach to the Neo-Hookean vessels and general shape vessels will be the subject of future investigations.

\section{Acknowledgements}

This work was partially supported by the Federal Program "Academic and pedagogical staff of innovative Russia", RAS program "Basic Research for Medicine", RFBR grants 08-01-00159, 09-01-00115, 10-01-91055.

\section{References}

[1] G. Cheng, H. Loree, R. Kamm, M. Fishbein, R. Lee. Distribution of circumferential stress in ruptured and stable atherosclerotic lesions: a structural analysis with histopathological correlation. Circulation, 87 (1993), 1179-1187. 
[2] L. Formaggia, A. Quarteroni, A. Veneziani. Cardiovascular mathematics, Vol. 1. Heidelberg, Springer, 2009.

[3] A. Green, J. Adkins. Large Elastic Deformation. Clarendon Press, Oxford, 1970.

[4] G. Holzapfel, R. Ogden (Eds.). Mechanics of Biological Tissue, Vol. XII. 2006.

[5] G. Holzapfel, R. Ogden. Constitutive modelling of arteries. Proc. R. Soc. A, 466 (2010), No. 2118, 1551-1597.

[6] J. Humphrey. Continuum biomechanics of soft biological tissues. Proc. R. Soc. Lond. A 459, (2003), 3-46.

[7] V. Koshelev, S. Mukhin, T. Sokolova, N. Sosnin, A. Favorski. Mathematical modelling of cardio-vascular hemodynamics with account of neuroregulation. Matem. Mod., 19 (2007), No. 3, 15-28 (in Russian).

[8] R. Lee, A. Grodzinsky, E. Frank, R. Kamm, F. Schoen. Structuredependent dynamic mechanical behavior of fibrous caps from human atherosclerotic plaques. Circulation, 83 (1991), 1764-1770.

[9] J. Ohayon et al. Influence of residual stress/strain on the biomechanical stability of vulnerable coronary plaques: Potential impact for evaluating the risk of plaque rupture. Am. J. Physiol. Heart Circ. Physiol. 293 (2007), 1987-1996.

[10] T.J. Pedley, X.Y. Luo. Modelling flow and oscillations in collapsible tubes. Theor. Comp. Fluid Dyn., 10 (1998), No. 1-4, 277-294.

[11] A. Quarteroni, L. Formaggia. Mathematical modelling and numerical simulation of the cardiovascular system. In: Handbook of numerical analysis, Vol.XII, Amsterdam, Elsevier, 2004, 3-127.

[12] W. Riley, R. Barnes, et al. Ultrasonic measurement of the elastic modulus of the common carotid. The Atherosclerosis Risk in Communities (ARIC) Study. Stroke, 23 (1992), 952-956.

[13] M. Rosar, C. Peskin. Fluid flow in collapsible elastic tubes: a three-dimensional numerical model. New York J. Math., 7 (2001), 281-302.

[14] S.S. Simakov, A.S. Kholodov. Computational study of oxygen concentration in human blood under low frequency disturbances. Mat. Mod. Comp. Sim., 1 (2009), 283-295.

[15] C. Tu, C. Peskin. Stability and instability in the computation of flows with moving immersed boundaries: a comparison of three methods. SIAM J. Sci. Stat. Comp., 6 (1992), No. 13, 1361-1376.

[16] Y.V. Vassilevski, S.S. Simakov, S.A. Kapranov. A multi-model approach to intravenous filter optimization. Int. J. Num. Meth. Biomed. Engrg., 26 (2010), No. 7, 915-925. 
[17] Y. Vassilevski, S. Simakov, V. Salamatova, Y. Ivanov, T. Dobroserdova. Blood flow simulation in atherosclerotic vascular network using fiber-spring representation of diseased wall. Math. Model. Nat. Phen. (in press), 2011.

[18] R. Vito, S. Dixon. Blood vessel constitutive models, 1995-2002. Annu. Rev. Biomed. Engrg., 5 (2003), 413-439.

[19] R. Wulandana. A nonlinear and inelastic constitutive equation for human cerebral arterial and aneurysm walls. Dissertation, University of Pittsburgh, Pittsburgh, 2003. 